TITLE:

\title{
Atomistic study of the competitive relationship between edge dislocation motion and hydrogen diffusion in alpha iron
}

\section{AUTHOR(S):}

Taketomi, Shinya; Matsumoto, Ryosuke; Miyazaki, Noriyuki

\section{CITATION:}

Taketomi, Shinya ... [et al]. Atomistic study of the competitive relationship between edge dislocation motion and hydrogen diffusion in alpha iron. JOURNAL OF MATERIALS RESEARCH 2011, 26(10): 1269-1278

ISSUE DATE:

2011-05

URL:

http://hdl.handle.net/2433/163441

RIGHT:

(c) Cambridge University Press 2011 


\title{
Atomistic study of the competitive relationship between edge dislocation motion and hydrogen diffusion in alpha iron
}

\author{
Shinya Taketomi ${ }^{\text {a) }}$ \\ Department of Mechanical Engineering, Graduate School of Science and Engineering, Saga University, \\ Saga 840-8502, Japan \\ Ryosuke Matsumoto and Noriyuki Miyazaki \\ Department of Mechanical Engineering and Science, Graduate School of Engineering, Kyoto University, \\ Kyoto 606-8501, Japan
}

(Received 9 December 2010; accepted 21 March 2011)

\begin{abstract}
The interaction between a dislocation and hydrogen is considered to play an important role in hydrogen-related fractures for metals; it has been experimentally reported that hydrogen affects the dislocation mobility. These studies, however, show different macroscopic softening and/or hardening effects in iron, and the interaction between the dislocation and hydrogen remains unclear. In this study, we investigated the occurrence of interactions between a $\{112\}<111>$ edge dislocation and a hydrogen atom via the estimation of the stress-dependent energy barriers for the dislocation motion and hydrogen diffusion in alpha iron using atomistic calculations. Our results show the existence of boundary stress conditions: dislocation mobility increment (softening) occurs at a lower applied stress, dislocation mobility decrement (hardening) occurs at an intermediate stress, and no effects occur for the steady motion of a dislocation at a higher stress in this analysis condition.
\end{abstract}

\section{INTRODUCTION}

The phenomenon of hydrogen affecting the mechanical property of metals, known as hydrogen embrittlement, is one of the fracture mechanisms that should be clarified for the safe use of structural materials. Many studies have been performed to clarify the fundamental effect of hydrogen on material strength to prevent hydrogen embrittlement. ${ }^{1-13}$ One of the typical effect of dissolved hydrogen appears on the plastic deformation behavior, which can be observed in the stress-strain relation and/or the fracture surface. ${ }^{13}$ Therefore, the dislocation motion in the presence of hydrogen is considered to play a fundamental role on hydrogen-related fractures for metals. ${ }^{5,6,9-11}$ For example, different effects of hydrogen on plastic deformation (macroscopic softening/hardening) have been reported in iron. ${ }^{14}$ These results show that the fundamental effect of hydrogen on plastic deformation is still unclear. Recently, much attention has been paid to the increment of the dislocation mobility (softening) as an elementary process for hydrogen embrittlement; this increment is known as the hydrogen-enhanced localized plasticity (HELP) mechanism., ${ }^{5,6}$ The HELP mechanism is considered to be responsible for the reduction of the energy barrier for dislocation motion ${ }^{5}$ or the hydrogen shielding effect due to the reduction of the interaction between dislocations. ${ }^{6}$ Sofronis et al. have reported that

\footnotetext{
a)Address all correspondence to this author.

e-mail: taketomi@me.saga-u.ac.jp

DOI: $10.1557 /$ jmr.2011.106
}

the hydrogen atmosphere shields the effective shear stress between dislocations under extremely high hydrogen concentrations. ${ }^{6}$ On the other hand, we reported that the energy barrier for $\{112\}<111>$ edge dislocation motion decreases in the presence of hydrogen in alpha iron using atomistic simulation. ${ }^{15}$ Our result indicates that hydrogen enhances the dislocation mobility due to the reduction of the energy barrier even in a low hydrogen concentration. However, our study estimated only the energy barrier for dislocation motion with a trapped hydrogen atom at the dislocation core; thus, the hydrogen diffusion accompanying the dislocation motion was not taken into consideration. The continuum interaction between the dislocation and the hydrogen atom is thought to occur when the frequency of the dislocation motion in the presence of hydrogen atom and the hydrogen diffusion process near the core are of similar magnitude (competitive relation). Moreover, hydrogen transportation by dislocation ${ }^{16}$ has been paid attention to as one of the hydrogen transportation mechanisms in metal. However, it is still difficult to observe the competition between the dislocation and hydrogen using experimental techniques as direct observation of the hydrogen atom is difficult. Hence, the estimation of the occurrence conditions of the competitive dynamic motion between the dislocation and hydrogen using computer simulation should be pivotal.

The interaction between a dislocation and a hydrogen atom is considered as one of the typical examples of the interaction between a dislocation and a solute atom. Investigation of the interaction between a dislocation and a solute atom has been conducted to clarify the solute 
effect on the dislocation motion (dislocation pinning/ unpinning at the solute atom). ${ }^{17,18}$ Particularly, dynamic strain aging (DSA) is paid much attention to as it attributes the competitive relationship between dislocation motion and solute diffusion. Pertaining to DSA, calculations based on continuum mechanics ${ }^{19-22}$ and atomistic models $^{23,24}$ have been adopted to calculate the dynamic effects of a solute atom on dislocation motion. To the extent of our knowledge, only one Monte Carlo simulation based on the continuum theory has been performed to estimate the interaction of a dislocation and hydrogen atom; ${ }^{25}$ however, this study could not take the dislocation core effect into consideration. Our previous study shows that hydrogen occupation within the dislocation core is the most stable. ${ }^{26}$ To consider the hydrogen atom trapped at the dislocation core, analysis based on an atomistic model is necessary. The classical molecular dynamics (MD) method is one of the powerful tools to calculate the atomic scale phenomena; however, the results could not be directly compared with the experimentally observed behavior due to the differences in timescale. Recently, the timescale gap has been tried to overcome by estimating activation energy by atomic scale simulations. ${ }^{24,27-29}$ These studies tried to connect the atomistic simulations of stress-driven processes to experimentally observed behavior via the estimation of stress-dependent activation energy barriers. These methods successfully predict the realistic timescale phenomenon compared with classical MD methods. Thus, we adopt these methods for the analysis of interaction between a dislocation and hydrogen atom.

Since the edge dislocation has the larger hydrostatic strain field compared with the screw dislocation, the edge dislocation has the strong interaction with hydrogen atoms. In this study, therefore, the $\{112\}<111>$ edge dislocation in alpha iron is examined. First, the hydrogen concentration around the dislocation core under a thermal equilibrium condition of hydrogen atmosphere is estimated based on the equilibrium theory and experimental results. Next, the correlation between the activation energy for the edge dislocation motion and the applied shear stress is investigated using the nudged elastic band (NEB) method ${ }^{30,31}$ to estimate the dislocation velocity in hydrogen-free alpha iron. Finally, the activation energy for the edge dislocation motion and hydrogen diffusion is calculated using the NEB method to estimate the occurrence conditions of the competitive relationship between the dislocation and hydrogen atom. Furthermore, the occurrence conditions of softening and/or hardening in the presence of hydrogen are also discussed.

\section{ANALYSIS METHODS}

\section{A. Analysis model}

The introduced $\{112\}<111>$ edge dislocation and the crystal orientation of the analysis model are shown in Fig. 1. The slip occurs on the $x z$-plane, and the slip direction [111] is set along the $x$-axis. To introduce an edge dislocation, an atomic plane with gray-colored atoms as shown in Fig. 1 was removed and then the relaxation of atomic structure was performed by the conjugate gradient (CG) method; this procedure is one of the molecular statics simulations; thus, the analysis temperature is $0 \mathrm{~K}$. During the structural relaxation process, the movement of atoms along the $y$-axis was fixed and then compression was applied along the $x$-axis to fill the removed plane. After that, the unit cell size and the atomic positions were relaxed until the $x$-axis stress $\left(\sigma_{x x}\right)$ decreased sufficiently $(\leq 0.005$ $\mathrm{MPa}$ ). The analysis system contains about 8000 iron atoms; the dimensions of the unit cell are 11.5, 4.9, and $2.0 \mathrm{~nm}$ along the $x-, y$-, and $z$-axes, respectively. Periodic boundary conditions were applied along the $x$ - and $z$-axes. Three atomic planes at the top and bottom of the unit cell perpendicular to the $y$-axis were considered as boundary planes and fixed. In this study, embedded atom method (EAM) potential developed by Wen et al. ${ }^{32}$ was adopted to describe the atomic interaction for the $\mathrm{Fe}-\mathrm{H}$ system.

\section{B. Hydrogen concentration around the dislocation core under hydrogen gaseous condition}

Many studies have been conducted on the hydrogen effect on alpha iron using an atomistic model, e.g., crack propagation $^{32}$ or dislocation motion. ${ }^{33,34}$ However, the hydrogen concentrations and/or distribution in these studies were based on assumptions. We, therefore, used more realistic hydrogen concentration and distribution around the $\{112\}<111>$ edge dislocation under a hydrogen gaseous condition using the hydrogen-trap energy distribution around the dislocation. ${ }^{26}$

According to Sievert's law, the equilibrium concentration of hydrogen is proportional to $p^{1 / 2}$ and $\exp (-\Delta H /$ $k_{\mathrm{B}} T$ ), where $p$ is the hydrogen gas pressure, $\Delta H$ is the heat of the solution, $k_{\mathrm{B}}$ is the Boltzman's constant, and $T$ is the temperature. Hirth ${ }^{35}$ has reported the hydrogen concentration (atom fraction of hydrogen) for alpha iron under the gaseous hydrogen condition based on Sievert's law. The hydrogen atom stably exists at the tetrahedral site (T-site)

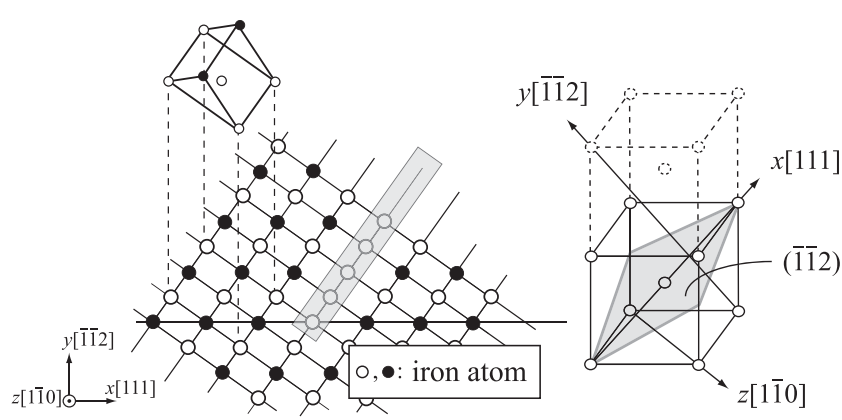

FIG. 1. Simulation model and crystal orientation of an $\{112\}<111>$ edge dislocation; an atomic plane with gray-colored atoms was removed and then the relaxation of atomic structure was performed to introduce an edge dislocation. 
within the nondeformed bcc-structured alpha iron. ${ }^{36}$ Therefore, the hydrogen occupancy at the T-site under thermal equilibrium conditions is given by Eq. (1) as a function of hydrogen gas pressure $p(\mathrm{~Pa})$ and temperature $T(\mathrm{~K})$.

$$
\theta_{\text {T-site }}=0.9686 \times 10^{-6} \sqrt{p} \exp \left(-\frac{3440}{T}\right) .
$$

Using the hydrogen occupancy at T-site, $\theta_{\mathrm{T} \text {-site, the }}$ hydrogen occupancy at a specific trap site $\theta_{i}$ with hydrogen-trap energy $E^{\text {Trap }}{ }_{i}$ under thermal equilibrium condition is given by the following equation: ${ }^{37}$

$$
\frac{\theta_{i}}{1-\theta_{i}}=\frac{\theta_{\mathrm{T} \text {-site }}}{1-\theta_{\mathrm{T}-\text { site }}} \exp \left(\frac{E^{\text {Trap }_{i}}}{k_{\mathrm{B}} T}\right),
$$

where the hydrogen-trap energy corresponds to the energy difference between the system with a hydrogen atom trapped at the specific trap site and the system with a hydrogen atom at a $\mathrm{T}$-site within the nondeformed perfect lattice; this definition is the same as that in our previous study. ${ }^{26}$ The correlation between hydrogen occupancy and hydrogen-trap energy for specific temperatures and gas pressures are shown in Fig. 2. In this figure, the value of hydrogen gas pressure is the maximum value of the expected vessel pressure (hydrogen gas tank for vehicles), i.e., $70 \mathrm{MPa}$ as shown in Fig. 2(a), and the temperature is $300 \mathrm{~K}$ as shown in Fig. 2(b). The maximum hydrogen-trap energy around the $\{112\}<111>$ edge dislocation is $0.49 \mathrm{eV}$ at the trap site within the dislocation core. ${ }^{26}$ Thus, a hydrogen atom is expected to be trapped at this strongest trap site with high probability at temperatures below $300 \mathrm{~K}$. The hydrogen occupancy is more sensitive to the hydrogen gas temperature compared with the hydrogen gas pressure as shown in Fig. 2.

Considering the structure of the edge dislocation, hydrogen-trap sites periodically exist along the dislocation line. The number of hydrogen atoms existing along a unit length of the dislocation line under a thermal equilibrium condition, neglecting hydrogen-hydrogen interaction, is approximately written as

$$
N_{\text {Total }}=\sum_{i=1} \theta_{i} n_{i},
$$

where $n_{i}$ is the number density of hydrogen trap sites per unit length with hydrogen-trap energy of $E^{\text {Trap }}{ }_{i}$. The total sum is taken for all the trap energies existing around the dislocation. To estimate the number of hydrogen atoms trapped around the dislocation under a thermalequilibrium condition, we take the hydrogen trap sites with the strongest trap energy $\left(E_{i}^{\text {Trap }}=0.49 \mathrm{eV}\right)$ into consideration for convenience. The probability of hydrogen existence is typically high at this trap site compared with other sites as shown in our previous study, ${ }^{15}$ and hence, this approximation is appropriate. Here, we introduced one hydrogen atom into the analysis model shown in Fig. 1, and thus the number of hydrogen atoms per unit length of dislocation line is $0.5 / \mathrm{nm}$, where cell size along $z$-direction is $\sim 2 \mathrm{~nm}$. The unit length of iron lattice along the dislocation line is $\sim 0.4 \mathrm{~nm}$ in this analysis model, i.e., the strongest hydrogen trap sites periodically exists every $0.4 \mathrm{~nm}$, and the number of the site is five in this analysis model. Therefore, the hydrogen occupancy should be 0.2 to realize the hydrogen concentration of $0.5 / \mathrm{nm}$. Using Eqs. (1) and (2), this hydrogen concentration $\left(C_{\mathrm{H}}=0.5 / \mathrm{nm}\right)$ under thermal equilibrium conditions can be obtained for different gas temperature and pressure as shown in Fig. 3. The curve shows that the analysis model adopted in this study contains hydrogen atoms as, for example, $300 \mathrm{~K}$ and $0.01 \mathrm{MPa}$ or $700 \mathrm{~K}$ and $100 \mathrm{MPa}$ hydrogen gaseous conditions at thermal equilibrium. The figure also shows the different hydrogen concentrations $\left(C_{\mathrm{H}}=0.01 / \mathrm{nm}\right)$, which will be discussed in following section.
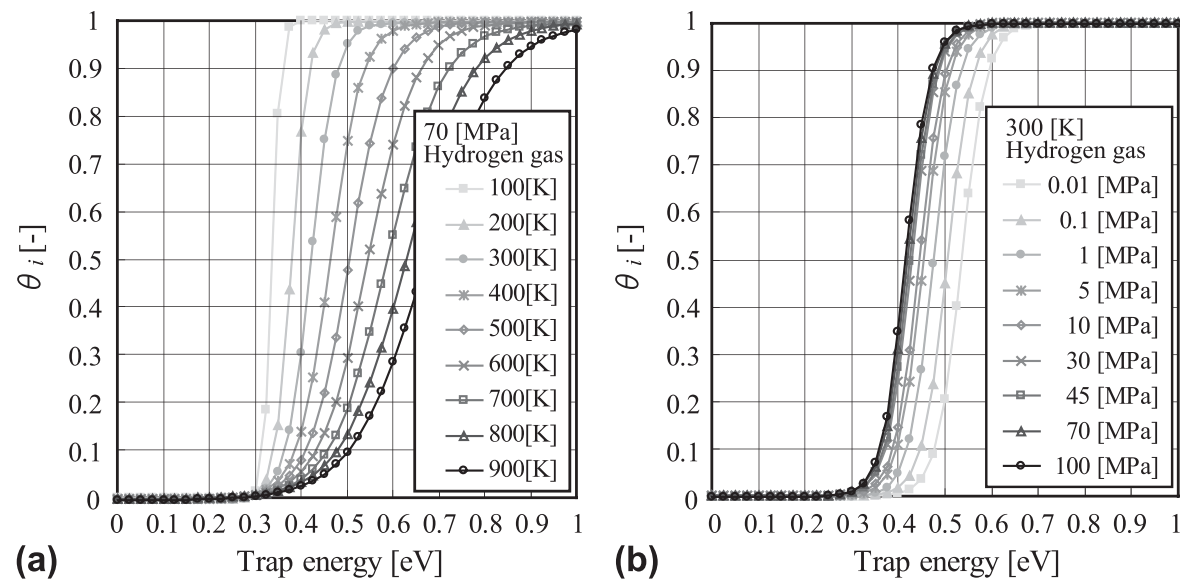

FIG. 2. Correlation between the hydrogen occupancy and hydrogen-trap energy; (a) effect of temperature with hydrogen gas pressure of $70 \mathrm{MPa}$ and (b) effect of hydrogen gas pressure with temperature of $300 \mathrm{~K}$. 


\section{ANALYTICAL RESULTS}

\section{A. Dislocation velocity in the absence of hydrogen}

First, the energy barrier for the dislocation-motion dependence on the applied shear stress in the absence of hydrogen is investigated using the NEB method; static calculation using CG method is adopted in this study. The NEB method enabled us to estimate the minimum energy path and the energy barrier for the reaction based on the given initial and final configurations. The corresponding displacements of the shear stress $\tau_{x y}$ are applied to the boundary atoms of the analysis model, then the positions of the other atoms are relaxed. The initial and final conditions of the NEB analyses are made with $1 b$ difference in the positions of the dislocation. The $\{112\}<111>$ edge dislocations have an anisotropic crystal structure with respect to the $y z$-plane; thus, there are two different (twinning and antitwinning) possible slip directions. In this study, the applied stress that derives dislocation moves along the antitwinning direction is arranged in the positive direction. Therefore, by increasing the applied stress, the dislocation tends to move along the antitwinning direction (right direction in Fig. 1).

Here, the potential energy of the system is revised as Eq. (4) to take the effect of the applied stress into consideration.

$$
\Phi_{\mathrm{F}}=\Phi-F \Delta x
$$

where $F$ is the force applied on the boundary atoms, $\Delta x$ is the displacement of the boundary atoms from the initial equilibrium positions, $\Phi_{\mathrm{F}}$ is the potential energy of the system that takes the work done by deformation into consideration, and $\Phi$ is the potential energy of the atomic system. The correlation between the shear stress and the energy barrier for the dislocation motion per unit length of

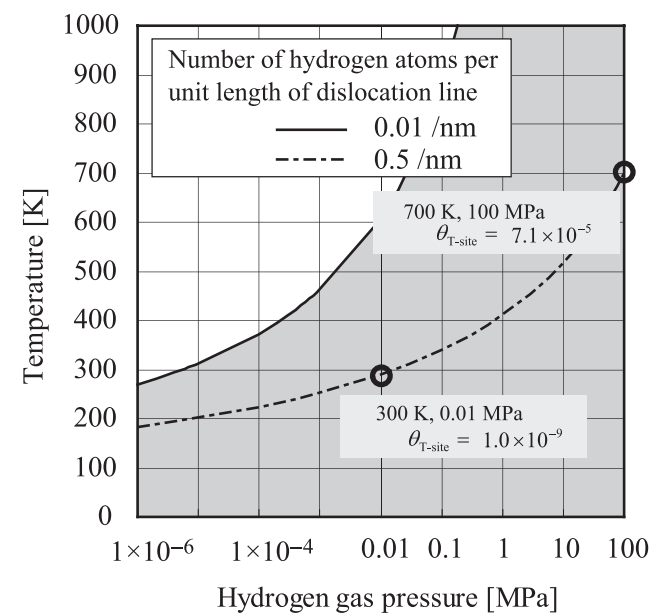

FIG. 3. Hydrogen concentration around the $\{112\}<111>$ edge dislocation at thermal equilibrium condition under hydrogen gaseous conditions. dislocation line along the antitwinning and twinning directions are shown in Fig. 4. Here, the energy barriers for the dislocation motion along each direction are written as $\Delta E_{\mathrm{w} / \mathrm{o}} \mathrm{H}$, anti-twin and $\Delta E_{\mathrm{w} / \mathrm{o}} \mathrm{H}$, twin. On increasing the applied stress, the differences between the energy barriers also increase.

In general, the edge dislocation motion is considered to occur with the kink-pair generation and migration under moderate stress as shown in Fig. 5(b). In this study, the direct evaluation of kink-pair generation and migration is not performed due to the limitation of the physical scale of the analysis model; i.e., when the applied stress is small, very large analysis models are required to form the kinks. Hence, the following approximated method is adopted. As the migration energy of kinks is usually small compared with that of the kink-pair generation, the dislocation motion is considered to be dominated by the kink-pair generation. Here, we assume the migration of the kinks is negligible, and the kink-pair generation is considered to be expressed as $V_{\mathrm{p}} \times l^{*}$, where $V_{\mathrm{p}}$ is the Peierls potential for edge dislocation motion and $l^{*}$ is the distance between kinks at the saddle point of the dislocation motion, which are required to surmount the energy barrier for dislocation motion as shown in Fig. 5(b). In addition, we assume that the edge dislocation moves $1 b$ in every step. In this study, Peierls potential dependence on applied stress is investigated using atomistic model and the distance between kinks, $l^{*}$ is given based on elastic dislocation theory as follows. ${ }^{38}$ The energy of the double-kinked segment in the dislocation is given by Eq. (5)

$$
F(x)=2 F_{\mathrm{k}}-\frac{\mu b^{4}}{8 \pi x}-\tau b^{2} x
$$

where $x$ is the separation distance between kinks, $b$ is the magnitude of burger's vector, $\mu$ is the shear modulus ( 68.6 GPa in this crystal orientation ${ }^{26}$ ), and $\tau$ is the applied shear stress. The first term corresponds to the energy of the kinks, second term is the elastic energy between kinks, and third term is the work done by applied stress. The separation distance between kinks at the saddle point of the dislocation motion derived from the differential of Eq. (5) becomes zero as shown in the following equation:

$$
\left[\frac{\partial F(x)}{\partial x}\right]_{x=l^{*}}=\frac{\mu b^{4}}{8 \pi x^{2}}-\tau b^{2}=0
$$

Thus,

$$
l^{*}=\left(\frac{\mu b^{2}}{8 \pi \tau}\right)^{1 / 2} .
$$

The correlation between the applied shear stress and the distance between kinks is shown in Fig. 5(a). On increasing the shear stress, the distance between kinks to 


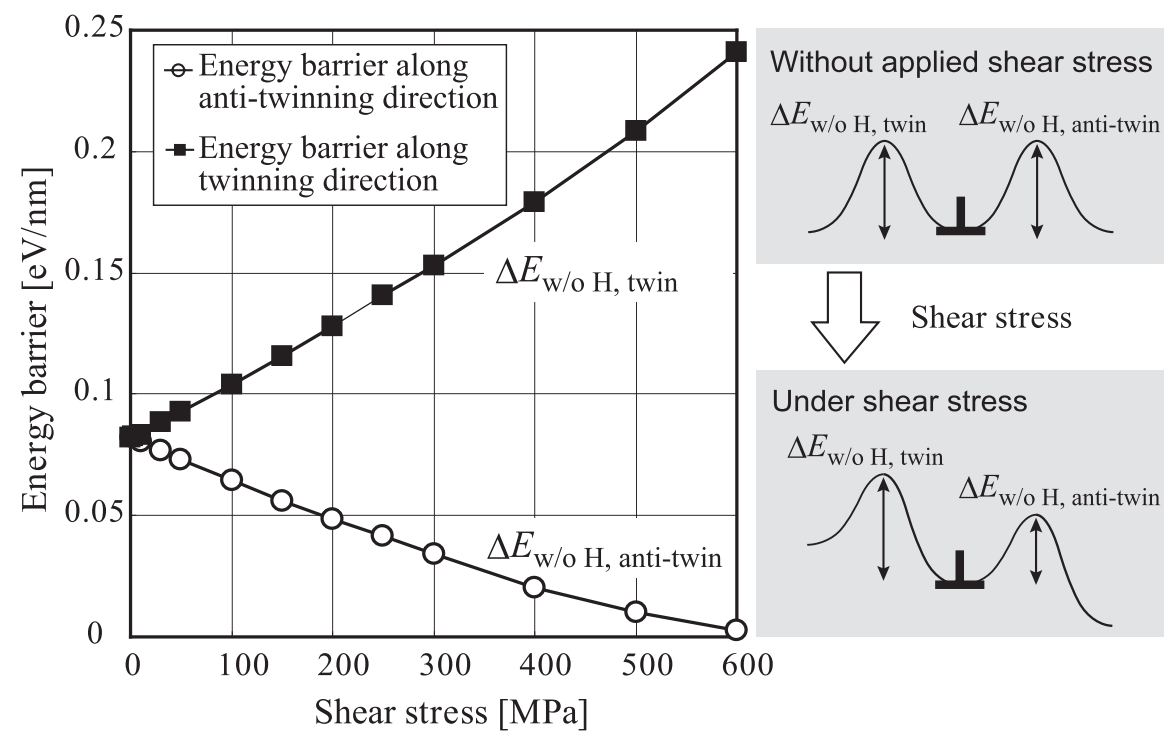

FIG. 4. Shear-stress dependence of the energy barrier for dislocation motion along antitwinning direction and twinning directions.

overtake the Peierls hill decreases. Here, upon increasing the applied stress, it is clear that the frequency of motion toward the twinning (negative) direction becomes very small compared to the antitwinning (positive) direction. Therefore, the dislocation motion toward the twinning direction is negligible. Furthermore, we assume that the energy of the kinks is negligibly small, and it leads to the conclusion that the edge dislocation velocity $v_{\mathrm{D}}$ can be given as

$$
v_{\mathrm{D}} \approx v_{\mathrm{D}, \text { anti-twin }}=Q\left(\Delta E_{\mathrm{w} / \mathrm{oH}, \text { anti-twin }} l^{*}\right) b
$$

where $Q(\Delta E)$ is the frequency to surmount the energy barrier of $\Delta E$. The dislocation motion is considered as a thermal activation process; thus, the frequency of motion is given as Eq. (9).

$$
Q(\Delta E)=v_{\mathrm{d}} \exp \left(-\frac{\Delta E}{k_{\mathrm{B}} T}\right),
$$

where $v_{\mathrm{d}}$ is the attempt frequency of the dislocation motion, $\Delta E$ is the energy barrier, and $k_{\mathrm{B}}$ is the Boltzman's constant. Here, the vibration frequency of the dislocation is given by the following equation:

$$
v_{\mathrm{d}} \approx v_{\mathrm{D}}\left(b / l^{*}\right)
$$

where $v_{D}$ is the Debye frequency and it is given as follows:

$$
v_{\mathrm{D}}=\left(\frac{18 \pi^{2} N}{\left(1 / s_{1}^{3}+2 / s_{\mathrm{t}}^{3}\right) V}\right)^{1 / 3}
$$

where $N$ is the number of iron atoms, $V$ is the volume; thus, $N / V$ corresponds to the number density of the iron

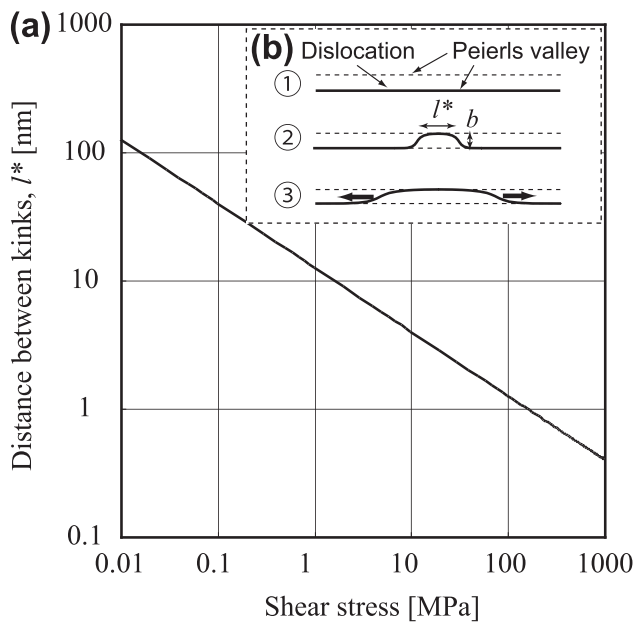

FIG. 5. Correlation between the applied shear stress and the distance between the kinks to overtake the Peierls potential.

crystal. $s_{1}$ and $s_{\mathrm{t}}$ are the longitudinal and transverse wave velocity and are obtained as 5950 and $3240 \mathrm{~m} / \mathrm{s}$ in iron, ${ }^{39}$ respectively. Substituting these values into Eq. (11), the Debye frequency of iron atoms as $0.62 \times 10^{14} \mathrm{~Hz}$ can be obtained.

The relationship between the dislocation velocity and the applied shear stress at $300 \mathrm{~K}$ is shown in Fig. 6. The dislocation velocity obtained in this study becomes faster than that of the elastic transverse wave in iron $(3240 \mathrm{~m} / \mathrm{s})$ at higher applied stress. According to the literature, ${ }^{40}$ a thermal activation process is considered to be dominant when the energy barrier of the thermal activation $\left(\Delta E_{\mathrm{w} / \mathrm{o}} \mathrm{H}\right.$, anti-twin $\left.{ }^{*}\right)$ is above $2 k_{\mathrm{B}} T$, and this condition is achieved below a stress of about $200 \mathrm{MPa}$. Therefore, stress conditions below $200 \mathrm{MPa}$ will be dealt with in this study. 


\section{B. Dislocation velocity in the presence of hydrogen}

\section{Conditions of dislocation traps hydrogen at thermal equilibrium}

The relationship of the relative positions between the dislocation and hydrogen can be characterized into three cases as shown in Fig. 7. Figure 7(a) is the condition in which no hydrogen atoms exist around the dislocation and the hydrogen atom can diffuse away. Figure 7(b) shows the condition in which the dislocation and the hydrogen atoms move in a state of competitive relationship. Figure 7(c) shows the condition in which only the dislocation moves away from the hydrogen atmosphere. To clarify the interactive condition between a dislocation and hydrogen, the occurrence conditions of Figs. 7(a)-7(c) are investigated.

First, the occurrence condition of Fig. 7(a) is clarified. This condition corresponds to the condition when hydrogen concentration is sufficiently low around a steady state dislocation without motion at thermal equilibrium. Here, we assumed the condition when the number of hydrogen atoms trapped by the dislocation becomes $0.01 / \mathrm{nm}$ as a representing example that corresponds to the hydrogen diffuse away condition as shown in Fig. 7(a). Using Eqs. (1) and (2), the conditions of hydrogen concentration of $0.01 / \mathrm{nm}$ for different temperatures and hydrogen gas pressures are obtained and are shown in Fig. 3. It shows that hydrogen atoms are assumed to diffuse away from the dislocation core under the condition as shown in the white region in Fig. 3.

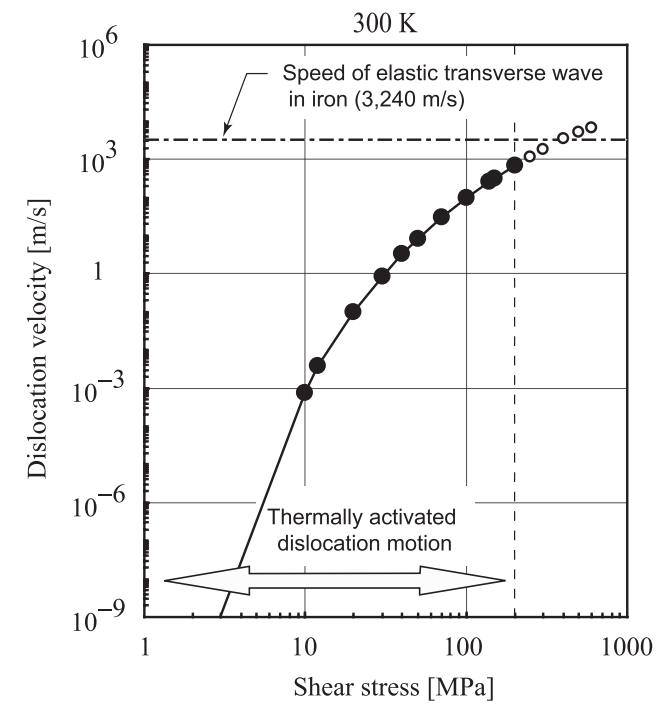

FIG. 6. Correlation between dislocation velocity and shear stress in the absence of hydrogen.

\section{Conditions of interaction between dislocation and hydrogen atom}

In this section, the occurrence conditions of interaction between a dislocation and hydrogen atom are clarified by estimating the occurrence conditions of Figs. 7(b) and 7(c). The analyses of the energy barrier for the dislocation motion in the presence of a hydrogen atom are performed using the NEB method. The energy barrier for the dislocation motion per unit length of the dislocation line in the presence of a hydrogen atom is shown in Fig. 8. Here, no shear stress is applied on the system. When the dislocation is positioned at $0 b$, a hydrogen atom is trapped at the trap site within the dislocation core with the strongest trap energy, where the potential energy of this condition is taken as zero. A typical effect of hydrogen on the energy barrier is observed while the $\{112\}<111>$ edge dislocation is located at $-3 b$ to $5 b$ from a hydrogen atom. This asymmetrical distribution of the potential energy is attributable to the crystallographic anisotropic structure of the $\{112\}<111>$ edge dislocation in alpha iron; this anisotropic effect clearly exists in the presence of a hydrogen atom. Here, the energy barrier of dislocation motion per unit length from $0 b$ to $1 b$ is defined as $\Delta E_{\mathrm{H}}$, $0 \rightarrow 1$. The other energy barriers are determined as shown in Fig. 8, where the condition of $\Delta E_{\mathrm{H}, 0 \rightarrow 1}<\Delta E_{\mathrm{w} / \mathrm{o} \mathrm{H} \text {, anti-twin }}$ $<\Delta E_{\mathrm{H}, 1 \rightarrow 2}$ is satisfied at the stress-free conditions.

Considering the dislocation motion in the presence of hydrogen, hydrogen atom diffusion must be considered. Only the dislocation motion along the antitwinning direction (positive direction of horizontal axis in Fig. 8) is taken into consideration as done in Sec. III. A. Here, the competitive motion between the dislocation and hydrogen atom can be characterized as (i) the hydrogen atom diffuses at first, then the dislocation follows the hydrogen atom, (ii) the hydrogen atom and the dislocation move at the same time, and (iii) the dislocation moves first, then the hydrogen atom diffuses to the dislocation. Pertaining to case (i), the hydrogen atom is initially trapped at the most stable occupation site, and the hydrogen atom is supposed to be retrapped at the same trap site even though hydrogen diffusion has occurred. Hence, case (i) is considered as hard to occur. Concerning case (ii), the NEB calculation shows that the simultaneous motion of the dislocation and the hydrogen atom needs to

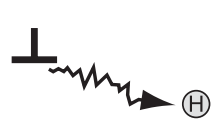

(a)

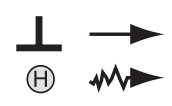

(b)

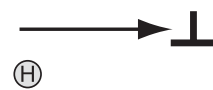

(c)
FIG. 7. Schematic of the relationship between a dislocation and a hydrogen atom; (a) dislocation does not trap the hydrogen atom and the hydrogen atom diffuses away from dislocation, (b) dislocation and hydrogen move at competitive velocities with interactions with each other, and (c) dislocation moves only ahead of hydrogen. 


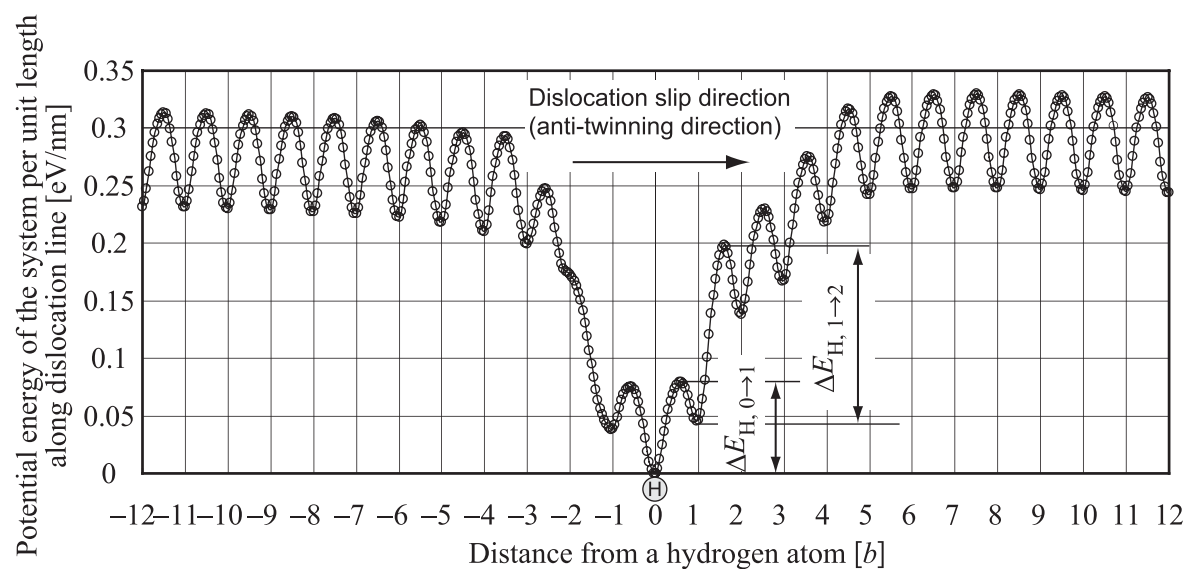

FIG. 8. Potential energy barrier of $\{112\}<111>$ edge dislocation motion around a hydrogen atom.

surmount extremely high energy barrier compared to the individual motion; $\sim 0.53$ and $0.08 \mathrm{eV} / \mathrm{nm}$ without applied stress, respectively, and hence, case (ii) is considered as hard to occur. Therefore, cases (i) and (ii) are considered negligible and only case (iii) is taken into consideration. The initial conditions of the analysis are written as follows: the dislocation initially surmounts the energy barrier of $\Delta E_{\mathrm{H}, 0 \rightarrow 1}$ and remains at $1 b$; in contrast, a hydrogen atom remains at $0 b$. The next event is whether the dislocation surmounts $\Delta E_{\mathrm{H}, 1 \rightarrow 2}$ or a hydrogen atom diffuses to chase the dislocation. The energy barrier of the minimum energy path (MEP) for hydrogen diffusion from the trap site located at approximately $1 b$ behind the dislocation core is

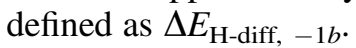

Therefore, the dislocation leaves the hydrogen atom as shown in Fig. 7(c) and can be realized under the conditions of

$$
Q\left(\Delta E_{\mathrm{H}-\mathrm{diff},-1 b}\right)<Q\left(\Delta E_{H, 1 \rightarrow 2} l^{*}\right) \quad,
$$

where the dislocation has to surmount the high energy barrier $\left(\Delta E_{\mathrm{H}, 1 \rightarrow 2}\right)$ compared with that in the absence of the hydrogen atom $(\Delta E$ w/o $\mathrm{H}$, anti-twin $)$ to leave the hydrogen atom at the initial position. Thus, the initial dislocation velocity becomes slow, after that the velocity is equal to the velocity in the absence of hydrogen as there is no interaction between the dislocation and hydrogen atom. Other than the above conditions, the dislocation and hydrogen atom move with interaction with each other. When

$$
Q\left(\Delta E_{\mathrm{H} \text {-diff, }-1 b}\right)<Q\left(\Delta E_{H, 0 \rightarrow 1} l^{*}\right),
$$

and

$$
Q\left(\Delta E_{H, 1 \rightarrow 2} l^{*}\right)<Q\left(\Delta E_{\mathrm{H}-\text { diff, }-1 b}\right),
$$

are satisfied, hydrogen diffusion dominates the dislocation velocity. In contrast, when

$$
Q\left(\Delta E_{H, 0 \rightarrow 1} l^{*}\right)<Q\left(\Delta E_{\mathrm{H}-\mathrm{diff},-1 b}\right),
$$

is satisfied, the hydrogen atom is supposed to have sufficient mobility to catch up to the dislocation soon after the dislocation motion, and the dislocation velocity is dominated by the frequency of $Q\left(\Delta E_{\mathrm{H}, 0 \rightarrow 1} l^{*}\right)$.

\section{Hydrogen effect on dislocation velocity}

Energy-barrier dependence on applied shear stress in the presence of hydrogen is investigated using the NEB method as explained in Sec. III. A. Here, we assume the $l^{*}$ derived from Eq. (7) does not change even in the presence of hydrogen, and one can obtain the frequency of motion for each energy barrier using Eqs. (9)-(11). Hydrogen diffusion can be treated as a thermal activation process similar to the dislocation motion. Thus, the frequency of diffusion can be written as

$$
Q=v \exp \left(-\frac{\Delta E}{k_{\mathrm{B}} T}\right),
$$

where $v$ is the attempt frequency of the hydrogen atom diffusion. Here, we adopt the frequency of hydrogen atoms as $1.11 \times 10^{14} \mathrm{~Hz}$; here we adopt the frequency of a pair hydrogen atoms obtained by the MD simulation using the same atomic potential as an approximated value. Pertaining to hydrogen diffusion, the MEP of hydrogen diffusion from $-1 b$ behind the edge dislocation core is investigated. The analysis result under stress-free conditions is shown in Fig. 9, where large gray spheres and small open circle correspond to iron atoms and hydrogen occupation sites, respectively. Hydrogen occupation sites are determined using Voronoi tessellation, and CG method is adopted for structural relaxation as in our previous study, ${ }^{26}$ therefore, all T- and O-sites around the dislocation core which can be detected by our procedure is taken into consideration. The iron atoms covered with a gray ellipse 
are non-bce structured atoms detected by common neighbor analysis, ${ }^{41}$ and this region is believed to be the dislocation core. The initial position of the hydrogen atom is assumed to be located at (1). The energy barriers of all the possible diffusion paths are investigated. As a result, the hydrogen diffusion path (black line in Fig. 9) is detected as the MEP. In addition, the maximum energy barrier of the MEP (bold line (1)-(2) in Fig. 9) is evaluated as $0.26 \mathrm{eV}$. According to our calculation, this energy barrier is almost independent of the applied shear stress. Therefore, we assume that the energy barrier of the hydrogen diffusion from the trap site located at $-1 b$ behind the dislocation is independent of the applied shear stress as $0.26 \mathrm{eV}$.

The dislocation motion frequency, $Q\left(\Delta E_{\mathrm{H}, 0 \rightarrow 1} l^{*}\right)$, $Q\left(\Delta E_{\mathrm{H}, 1 \rightarrow 2} l *\right), Q\left(\Delta E_{\mathrm{w} / \mathrm{o} \mathrm{H} \text {, antit-win }} l^{*}\right)$, and the hydrogen diffusion frequency $Q\left(\Delta E_{\mathrm{H}-\text { diff, }}-1 b\right)$ are compared as a function of shear stress as shown in Fig. 10. The result at 300 and $700 \mathrm{~K}$ are shown in Figs. 10(a) and 10(b), respectively. In this figure, $\tau_{\mathrm{I}}$ and $\tau_{\mathrm{II}}$ are the shear

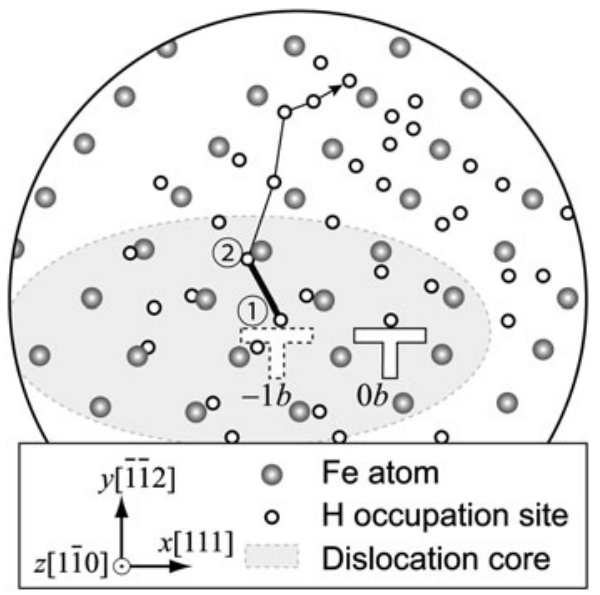

FIG. 9. Distributions of iron atoms and hydrogen trap sites around the dislocation core, and minimum energy path of hydrogen diffusion from $1 b$ behind the dislocation core. stresses that satisfy the conditions of $Q\left(\Delta E_{\mathrm{H}, 0 \rightarrow 1} l^{*}\right)=$ $Q\left(\Delta E_{\mathrm{H} \text {-diff, }-1 b}\right)$ and $Q\left(\Delta E_{\mathrm{H}, 1 \rightarrow 2} l^{*}\right)=Q\left(\Delta E_{\mathrm{H} \text {-diff, }-1 b}\right)$, respectively, and $\tau_{\mathrm{s}-\mathrm{h} \text { trans }}$ is the shear stress that satisfies the condition of $Q\left(\Delta E_{\mathrm{w} / \mathrm{o} \mathrm{H} \text {, anti-twin }} l^{*}\right)=Q\left(\Delta E_{\mathrm{H} \text {-diff, }-1 b}\right)$. When the applied shear stress goes below $\tau_{\mathrm{I}}$, it satisfies Eq. (15). Therefore, the dislocation motion is dominated by $Q\left(\Delta E_{\mathrm{H}, 0 \rightarrow 1} l^{*}\right)$, and the dislocation velocity is written as $v_{\mathrm{D}}^{\mathrm{H}}=Q\left(\Delta E_{\mathrm{H}, 0 \rightarrow 1} l^{*}\right) b$. When the applied shear stress is larger than $\tau_{\mathrm{I}}$ and smaller than $\tau_{\mathrm{II}}$, Eqs. (13) and (14) are satisfied. Therefore, the dislocation motion is dominated by the hydrogen diffusion frequency $Q\left(\Delta E_{\mathrm{H}-\mathrm{diff}},-1 b\right)$ and the dislocation velocity is written as $v_{\mathrm{D}}^{\mathrm{H}}=Q\left(\Delta E_{\mathrm{H}-\mathrm{diff},-1 \mathrm{~b}}\right) b$. Within this condition, the dislocation velocity becomes faster than that in the absence of hydrogen when the applied shear stress is smaller than $\tau_{\mathrm{s}-\mathrm{h}}$ trans; in contrast, the dislocation velocity becomes slower when the applied shear stress is larger than $\tau_{\mathrm{s}-\mathrm{h} \text { trans. }}$. On the other hand, the dislocation escapes from hydrogen when the applied shear stress is larger than $\tau_{\mathrm{II}}$. In this case, the dislocation velocity at the very initial state is slower than that in the absence of hydrogen due to the dislocation having to surmount the higher energy barrier $\left(\Delta E_{\mathrm{H}, 1 \rightarrow 2}\right)$. However, after that the dislocation velocity becomes the same as that in the absence of hydrogen. The correlation between the dislocation velocity and the applied shear stress is shown in Fig. 11. The results clearly show that softening occurs when the shear stress goes below $\tau_{\mathrm{s}-\mathrm{h} \text { trans }}$, and hardening occurs when the shear stress is in the region between $\tau_{\mathrm{s}-\mathrm{h} \text { trans }}$ and $\tau_{\mathrm{II}}$ in these analyses conditions. Moreover, the correlation between the increment of the dislocation velocity, $v_{\mathrm{D}}^{\mathrm{H}} / v_{\mathrm{D}}$, and the applied shear stress is shown in Fig. 12. It shows that by decreasing the stress, the magnitude of the softening increases in the softening region, which is qualitatively in good agreement with the experimental results. ${ }^{5}$ And by increasing the stress, the magnitude of the hardening increases in the hardening region.
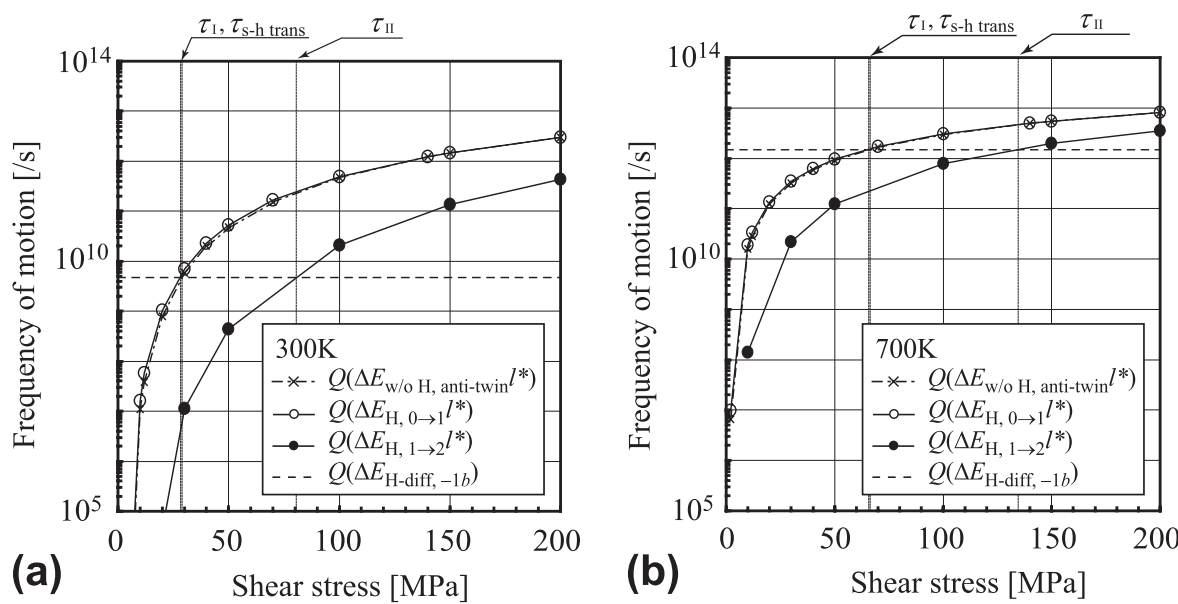

FIG. 10. Correlation between frequency of motion and applied shear stress. 

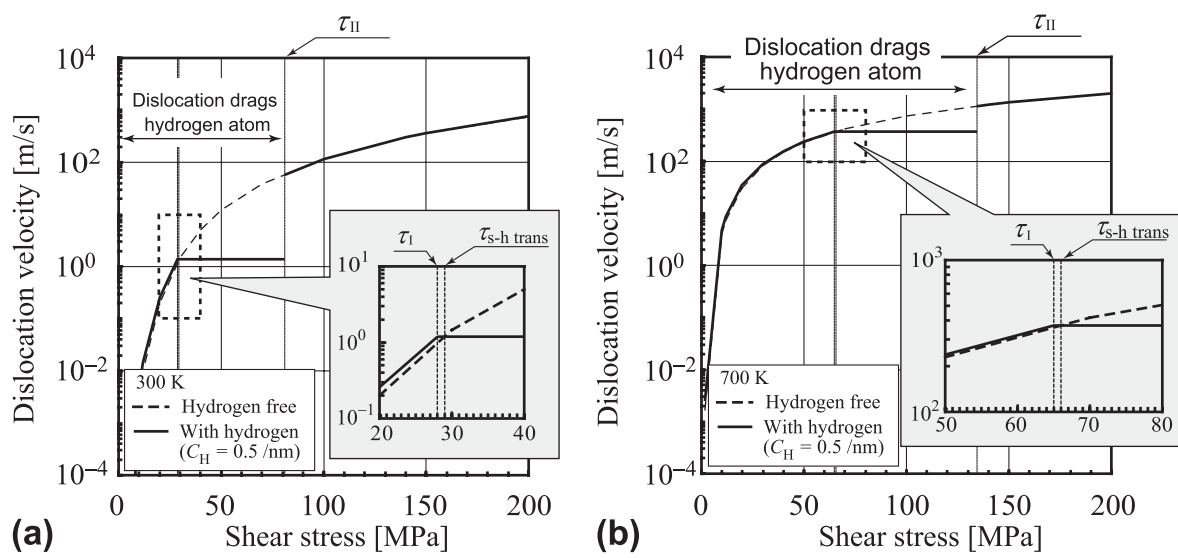

FIG. 11. Correlation between shear stress and dislocation velocity in the presence of hydrogen: (a) $300 \mathrm{~K}$ and (b) $700 \mathrm{~K}$.

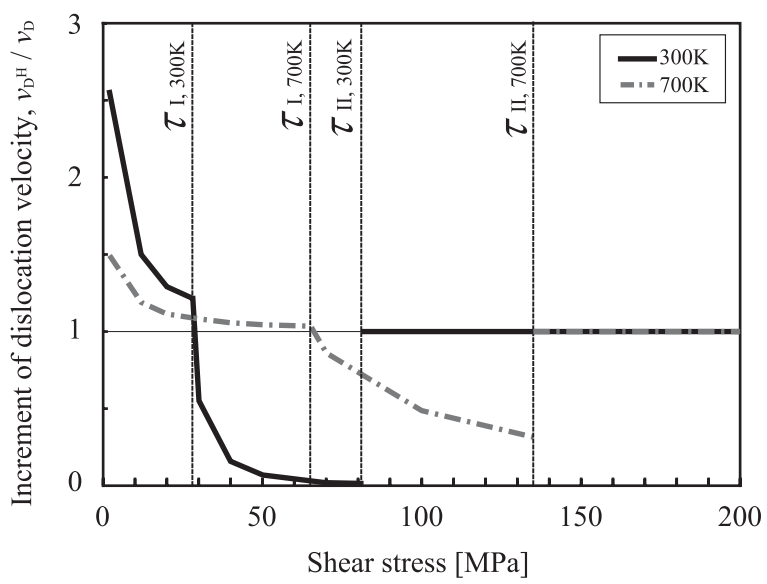

FIG. 12. Increment of dislocation velocity, $v_{\mathrm{D}}^{\mathrm{H}} / \boldsymbol{v}_{\mathrm{D}}$, in the presence of hydrogen.

Our results indicate that the dislocation mobility in the presence of hydrogen varies from softening to hardening depending on the applied shear stress. This leads to the conclusion that the dislocation motion around a crack shows characteristic behavior in a hydrogen environment. This indicates that an increment of the dislocation mobility in the presence of hydrogen occurs near the crack with a lower stress intensity factor. In this condition, the effective shear stress around the crack will be small and results in the acceleration of the plastic deformation. On the other hand, if the stress intensity factor becomes sufficiently large, then the dislocation mobility decreases compared with that in the absence of hydrogen. Thus, the plastic deformation is interrupted, and the distribution of the dislocations localizes around the crack. In fact, the hydrogen environment enhancement of the dislocation distribution localization around a crack was observed around the higher stress intensity factor crack $^{42,43}$ and complex distribution at lower stress intensity factor conditions ${ }^{44}$ using transmission electron microscopy for Fe-3.2 wt $\%$ Si.

\section{CONCLUSIONS}

We investigated the occurrence conditions of the interaction between the $\{112\}<111>$ edge dislocation and a solute hydrogen atom $\left(C_{\mathrm{H}}=0.5 / \mathrm{nm}\right)$ in alpha iron via the estimation of the stress-dependent activation barriers of the dislocation motion and hydrogen diffusion. After the classification of the dislocation motion and hydrogen diffusion, the correlation between the dislocation velocity and the applied shear stress was estimated. The results show that the increment of the dislocation velocity (softening) occurs when the applied stress is lower than $\tau_{\mathrm{s}-\mathrm{h} \text { trans. }}$. The decrement of the dislocation velocity (hardening) occurs when the applied stress is larger than $\tau_{\mathrm{s}-\mathrm{h} \text { trans }}$ and smaller than $\tau_{\mathrm{II}}$. In these conditions $\left(\tau<\tau_{\mathrm{II}}\right)$, the dislocation and hydrogen atom have an interaction with each other and result in competitive motion. In contrast, when the applied shear stress is larger than $\tau_{\text {II }}$, the dislocation leaves the hydrogen atom.

\section{ACKNOWLEDGMENT}

This research was partially supported by the Fundamental Research Project on Advanced Hydrogen Science funded by the New Energy Development Organization (NEDO).

\section{REFERENCES}

1. R.P. Frohmberg, W.J. Barnett, and A.R. Troiano: Delayed failure and hydrogen embrittlement in steel. Trans. ASM 47, 892 (1955).

2. R.A. Oriani and H. Josephic: Equilibrium aspects of hydrogeninduced cracking of steels. Acta Metall. 22, 1065 (1974).

3. R.A. Oriani and H. Josephic: Equilibrium and kinetic studies of the hydrogen-assisted cracking of steel. Acta Metall. 25, 979 (1977).

4. C.D. Beachem: A new model for hydrogen-assisted cracking (hydrogen "embrittlement"). Metall. Trans. 3A, 437 (1972).

5. H.K. Birnbaum and P. Sofronis: Hydrogen-enhanced localized plasticity - a mechanism for hydrogen-related fracture. Mater. Sci. Eng., A 176, 191 (1994).

6. P. Sofronis and H.K. Birnbaum: Mechanics of the hydrogendislocation-impurity interactions: Part I increasing shear modulus. J. Mech. Phys. Solids 43, 49 (1995). 
7. M. Nagumo, M. Nakamura, and K. Takai: Hydrogen thermal desorption relevant to delayed-fracture susceptibility of highstrength steel. Metall. Mater. Trans. A 32A, 339 (2001).

8. K. Takai, H. Shoda, H. Suzuki, and M. Nagumo: Lattice defects dominationg hydrogen-related failure of metals. Acta Mater. 56, $5158(2008)$

9. S.P. Lynch: Environmentally assisted cracking overview of evidence for an adsorption-induced localised-slip process. Acta Metall. 36, 2639 (1988).

10. Y. Murakami and $H$. Matsunaga: The effect of hydrogen on fatigue properties of steels used for fuel cell system. Int. J. Fatigue 28, 1509 (2006).

11. Y. Murakami, T. Kanezaki, Y. Mine, and S. Matsuoka: Hydrogen embrittlement mechanism in fatigue of austenitic stainless steels. Metall. Mater. Trans. A 39A, 1327 (2008).

12. S.M. Myers, M.I. Baskes, H.K. Birnbaum, J.W. Corbett, G.G. Deleo, S.K. Estreicher, E.E. Haller, P. Jena, N.M. Johnson, R. Kirchheim, S.J. Pearton, and M.J. Stavola: Hydrogen interactions with defects in crystalline solids. Rev. Mod. Phys. 64, 559 (1992).

13. G. Han, J. He, S. Fukuyama, and K. Yokogawa: Effect of straininduced martensite on hydrogen environment embrittlement of sensitized austenitic stainless steels at low temperatures. Acta Mater. 46, 4559 (1998).

14. M. Nagumo: Fundamentals of Hydrogen Embrittlement (Uchida Rokakuho, Japan, 2008) p. 167. (in Japanese).

15. S. Taketomi, R. Matsumoto, and N. Miyazaki: Atomistic simulation of the effects of hydrogen on the mobility of edge dislocation in alpha iron. J. Mater. Sci. 43, 1166 (2008).

16. A.H. Cottrell: Dislocations and Plastic Flow in Crystals (Oxford University Press, England, 1953) p. 136.

17. A.H. Cottrell, and B.A. Bilby: Dislocation theory of yielding and strain ageing of iron. Proc. Phys. Soc. Sec. A 62, 49 (2949).

18. K. Tapasa, Y.N. Osetsky, and D.J. Bacon: Computer simulation of interaction of an edge dislocation with a carbon interstitial in alphairon and dffects on glide. Acta Mater. 55, 93 (2007).

19. S.Y. Hu, Y.L. Li, Y.X. Zheng, and L.Q. Chen: Effect of solutes on dislocation motion -a phase- field simulation. Int. J. Plast. 20, 403 (2004).

20. S.Y. Hu, J. Choi, Y.L. Li, and L.Q. Chen: Dynamic drag of solute atmosphere on moving edge dislocations-phase-field simulation. J. Appl. Phys. 96, 229 (2004).

21. Z.J. Chen, Q.C. Zhang, and X.P. Wu: Dynamic interaction between dislocation and diffusing solutes. Europhys. Lett. 71, 235 (2005)

22. J.M. Rickman, R. LeSar, and D.J. Srolovitz: Solute effects on dislocation glide in metals. Acta Mater. 51, 1199 (2003).

23. W.A. Curtin, D.L. Olmsted, and L.G. Hector Jr.: A predictive mechanism for dynamic strain ageing in aluminum-magnesium alloys. Nat. Mater. 5, 875 (2006).

24. D.L. Olmsted, L.G. Hector Jr., and W.A. Curtin: Molecular dynamics study of solute strengthening in $\mathrm{Al} / \mathrm{Mg}$ alloys. J. Mech. Phys. Solids 54, 1763 (2006).

25. Y. Wang, D.J. Srolovitz, J.M. Rickman, and R. LeSar: Dislocation motion in the presence of diffusing solutes: A computer simulation study. Acta Mater. 48, 2163 (2000).
26. S. Taketomi, R. Matsumoto, and N. Miyazaki: Atomistic study of hydrogen distribution and diffusion around a $\{112\}<111>$ edge dislocation in alpha iron. Acta Mater. 56, 3761 (2008).

27. T. Zhu, J. Li, A. Samanta, A. Leach, and K. Gall: Temperature and strain-rate dependence of surface dislocation nucleation. Phys. Rev. Lett. 100, 025502 (2008).

28. M. Wen, B. An, S. Fukuyama, K. Yokogawa, and A.H.M. Ngan: Thermally activated model for tensile yielding of pristine singlewalled carbon nanotubes with nonlinear elastic deformation. Carbon 47, 2070 (2009).

29. D.H. Warner and W.A. Curtin: Origins and implications of temperature dependent activation energy barriers for dislocation nucleation in fcc metals. Acta Mater. 57, 4267 (2009).

30. G. Henkelman and H. Jónsson: Improved tangent estimate in the nudged elastic band method for finding minimum energy paths and saddle points. J. Chem. Phys. 113, 9978 (2000).

31. H. Jónsson, G. Mills, and K.W. Jacobsen: Nudged elastic band method for finding minimum energy paths of transitions, in Classical and Quantum Dynamics in Condensed Phase Simulations, edited by B.J. Berne, G. Ciccotti, and D.F. Coker (World Scientific, Singapore, 1998), p. 385.

32. M. Wen, X.J. Xu, S. Fukuyama, and K. Yokogawa: Embeddedatom-method functions for the body-centered-cubic iron and hydrogen. J. Mater. Res. 16, 3496 (2001).

33. M. Wen, S. Fukuyama, and K. Yokogawa: Atomistic simulations of effect of hydrogen on kink-pair energetics of screw dislocations in bcc iron. Acta Mater. 51, 1767 (2003).

34. S. Nedelcu and P. Kizler: Molecular dynamics simulation of hydrogen-edge dislocation interaction in bcc iron. Phys. Status Solidi A 193, 26 (2002).

35. J.P. Hirth: Effects of hydrogen on the properties of iron and steel. Metall. Trans. A 11A, 861 (1980).

36. R. Matsumoto, Y. Inoue, S. Taketomi, and N. Miyazaki: Influence of shear strain on the hydrogen trapped in bcc-Fe: A first-principlesbased study. Scr. Mater. 60, 555 (2009).

37. R.A. Oriani: The diffusion and trapping of hydrogen in steel. Acta Metall. 18, 147 (1970).

38. J.P. Hirth and J. Lothe: Theory of dislocations, 2nd ed. (Krieger Publishing Company, 1982), p. 541.

39. National Astronomical Observatory: Chronological Science Tables (Maruzen Co., Ltd., Japan, 2003) (in Japanese).

40. T. Yokobori: Zairyo-Kyodo-Gaku (Gihodo Press, 1955) p. 14 (in Japanese).

41. J.D. Honeycutt and H.C. Andersen: Molecular dynamics study of melting and freezing of small lennard-jones clusters. J. Phys. Chem. 91, 4950 (1987).

42. Y. Takahashi, M. Tanaka, K. Higashida, and H. Noguchi: Hydrogeninduced slip localization around a quasi-brittle fatigue crack observed by high-voltage electron microscopy. Scr. Mater. 61, 145 (2009).

43. Y. Takahashi, M. Tanaka, K. Higashida, K. Yamaguchi, and H. Noguchi: An intrinsic effect of hydrogen on cyclic slip deformation around a $\{110\}$ fatigue crack in Fe-3.2 wt\% Si alloy. Acta Mater. 58, 1972 (2010).

44. Y. Takahashi, J. Sakamoto, M. Tanaka, K. Higashida, and H. Noguchi: TEM observation of cyclic deformation around an oblique fatigue crack tip in single-crystalline Fe-3.2 wt\% Si alloy. Trans. Japan Soc. Mech. Eng. A. 76, 251 (2010) (in Japanese). 